\title{
Approximate bounds and temperature dependence of adiabatic connection integrands for the uniform electron gas
}

\author{
Brittany P. Harding, ${ }^{1}$ Zachary Mauri, ${ }^{1}$ and Aurora Pribram-Jones ${ }^{1}$ \\ University of California, Merced, 5200 North Lake Road, Merced, CA 95343
}

(*Electronic mail: apj@ucmerced.edu)

(Dated: 24 November 2021)

Thermal density functional theory is commonly used in simulations of warm dense matter, a highly energetic phase characterized by substantial thermal effects and by correlated electrons demanding quantum mechanical treatment. The numerous approximations for the exchange-correlation energy component in zero-temperature density functional theory, though often used in these high-energy-density simulations with Fermi-weighted electronic densities, are known to miss temperature-dependent effects in the electronic structure of these systems. In this work, the temperaturedependent adiabatic connection is demonstrated and analyzed using a well-known parameterization of the uniform electron gas free energy. Useful tools based on this formalism for analyzing and constraining approximations of the exchange-correlation at zero temperature are leveraged for the finite-temperature case. Inspired by the Lieb-Oxford inequality, which provides a lower bound for the ground-state exchange-correlation energy, bounds for the exchangecorrelation at finite temperatures are approximated for various degrees of electronic correlation.

\section{INTRODUCTION}

Recent decades have seen a rapid growth of interest in the study of matter under conditions of extreme excitation and/or compression. This interest includes growing focus on warm dense matter (WDM), a highly energetic phase characterized by the simultaneous existence of stronglycorrelated electrons, temperature effects, and quantum effects of electrons. ${ }^{1-5}$ Astrophysical examples of WDM include white dwarf atmospheres and planetary cores, ${ }^{6,7}$ while more down to earth examples include laser-excited solids and inertial confinement fusion capsules. ${ }^{8-10}$ There have been corresponding advances in the use of density functional theory ${ }^{11,12}$ (DFT) calculations of WDM. Aside from describing planetary interiors, ${ }^{13-15}$ these calculations are useful for predicting material properties, ${ }^{4,5,16}$ developing experimental standards, ${ }^{3,17}$ and supplementing experiments that push the boundaries of accessible conditions. ${ }^{18-21}$

In almost all thermal DFT calculations, a small-but-crucial free energy component, called the exchange-correlation (XC), is approximated by a ground-state approximation. However, in principle, the $\mathrm{XC}$ free energy depends explicitly on the temperature. $^{22-25}$ If the exact temperature-dependent XC free energy were known, the free energy and density for a given distribution of the nuclei, and any properties that could be extracted from these, such as Hugoniot shock curves and equations of state of materials, could be predicted exactly. ${ }^{26}$ While using a ground-state approximation to the $\mathrm{XC}$ free energy has not been shown to be a fatal flaw, the pervasive use of this approximation is a continuing source of concern that warrants investigation. $4,5,25,27-29$

Approximations to the XC functional can be informed by the adiabatic connection formalism, which is an important analytical and interpretive tool in DFT. ${ }^{30-32}$ The adiabatic connection formula expresses $E_{\mathrm{XC}}[n]$ exactly as an integral over the coupling constant, $\lambda$, which smoothly connects the fictitious non-interacting Kohn-Sham reference system $(\lambda=0)$ with the real physical interacting system $(\lambda=1)$, while hold- ing the density fixed. A similar theoretical construction with explicit temperature dependence for an ensemble at finite temperature exists, ${ }^{25}$ but it has only been numerically demonstrated as it has for zero-temperature systems using model systems such as the Hubbard dimer. ${ }^{28,33}$ )

A useful tool in the quest for better functional approximations is the list of known exact properties and constraints on the formally exact XC functional. ${ }^{34,35}$ The LO bound ${ }^{36-43}$ is an exact inequality that provides a strict bound on the $\mathrm{XC}$ energy, and it has been used to constrain some of the most successful XC approximations. Perdew derived looser lower bounds on the $\mathrm{XC}$ and exchange energies using the LO lower bound on the indirect Coulomb energy, ${ }^{44}$ which was subsequently used in the development of the PerdewBurke-Ernzerhof ${ }^{45}$. While GGAs must violate this constraint for realistic energetic predictions, ${ }^{46}$ a meta-GGA that satisfies important constraints, including the LO bound, was constructed by Sun and collaborators ${ }^{47}$. The few temperaturedependent XC approximations developed in the literature ${ }^{48}$ are constructed to satisfy the LO bound, but the effect of temperature on this formal relationship has not been explored in a detailed manner.

In this work, finite-temperature adiabatic connection (FT AC) curves are generated using a parameterization ${ }^{49}$ of the $\mathrm{XC}$ free energy of the uniform electron gas (UEG) for various temperatures and densities in the WDM regime. In order to validate the finite-temperature simulated scaling approach used in this work, calculated $\mathrm{XC}$ free energy values are compared to $\mathrm{XC}$ free energy values generated from the Fortran 90 program provided by Groth and collaborators, ${ }^{49}$ upon which the simulated scaling results also rely. In addition, the behavior of simulated curves is evaluated in light of known mathematical conditions on the exchange free energy. The calculated adiabatic connection curves are used to explore how the balance between exchange and correlation changes in different regimes. Subsequently, adherence to the LiebOxford (LO) bound is analyzed and an approach to estimating temperature-dependence is evaluated. Approximate bounds are generated numerically using these results and compared 
to those known at ZT.

\section{BACKGROUND}

Hohenberg and Kohn have shown ${ }^{11}$ that there exists a functional of the density such that

$$
E \equiv \int d \mathbf{r} v(\mathbf{r}) n(\mathbf{r})+F[n(\mathbf{r})]
$$

is equal to the ground-state energy when $n(\mathbf{r})$ is the groundstate density. ${ }^{11}$ DFT relies on the fact that the ground-state energy is expressed as a functional of the ground-state density. The core idea of Kohn-Sham DFT, ${ }^{12}$ one of the most successful approaches to the many-body problem, is to approach the interacting problem of interest by mapping it to a more tractable non-interacting problem. This mapping would be exact if the correct exchange-correlation (XC) functional for arbitrary physical systems was available. However, this is not the case in the vast majority of systems, and so one must use approximations for practical calculations.

The zero-temperature (ZT) adiabatic connection gives an exact expression for the exchange-correlation functional,

$$
E_{\mathrm{XC}}[n]=\int_{0}^{1} d \lambda W_{\mathrm{XC}}^{\lambda}[n]
$$

expressed as an integral over a coupling constant $\lambda$, which, while holding the density $n(\mathbf{r})$ fixed, continuously connects the KS reference system $(\lambda=0)$ with the real physical interacting system $(\lambda=1) .{ }^{30-32}$ The integrand, $W_{\mathrm{xC}}^{\lambda}[n]$, is generated by introducing $\lambda$ into the universal functional, $F^{\lambda}[n]=$ $\min _{\Psi \rightarrow n}\left\langle\Psi\left|\hat{T}+\lambda \hat{V}_{e e}\right| \Psi\right\rangle$, where $\Psi$ is the minimizing wave function for a given $\lambda$. For all values of $\lambda$, the density remains that of the real physical system (the superscript $\lambda$ is often omitted when $\lambda=1$ ). An illustration of the adiabatic connection, which provides a geometrical representation of the components of $E_{\mathrm{XC}}$, is shown in Fig. 1.

Moving to non-zero temperatures requires shifting our focus from ground-state energy functionals to free energy functionals. Mermin ${ }^{22}$ generalized the Hohenberg-Kohn (HK) theorems to equilibrium systems at finite temperatures. At non-zero temperatures, many-electron systems can be described by the grand-canonical ensemble. The grand potential ${ }^{25}$ can be written,

$$
\hat{\Omega}=\hat{H}-\tau \hat{S}-\mu \hat{N},
$$

where $\hat{H}$ represents the electronic Hamiltonian operator, $\tau$ the temperature, $\hat{S}$ the entropy operator, $\mu$ the chemical potential, and $\hat{N}$ the particle-number operator. The entropy operator is defined via,

$$
\hat{S}=-k_{B} \ln \hat{\Gamma}
$$

where $\hat{\Gamma}$ is the statistical operator,

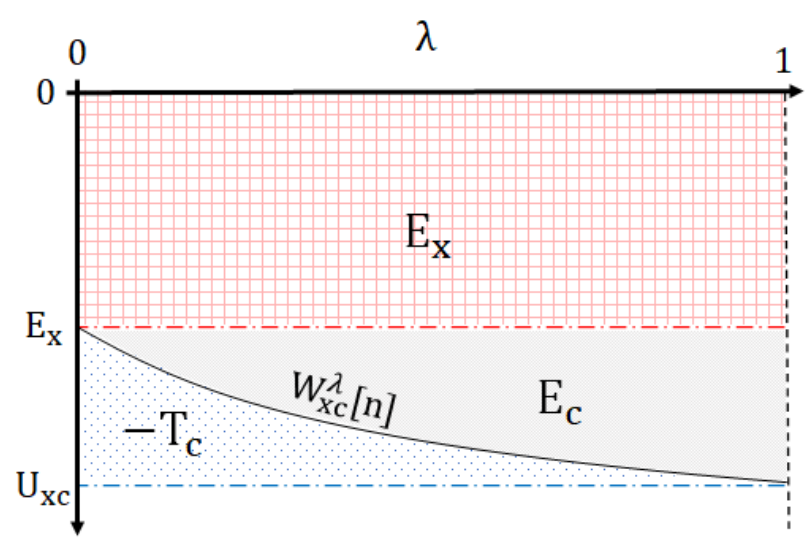

FIG. 1. A diagram of the adiabatic connection at zero temperature. The area in red, $E_{\mathrm{X}}$, represents the exchange energy component of $E_{\mathrm{XC}}$, while the gray area, $E_{\mathrm{C}}$, depicts the correlation energy component. The blue shaded region is related tot he kinetic correlation component of $E_{\mathrm{XC}}$.

$$
\hat{\Gamma}=\sum_{N, i} w_{N, i}\left|\Psi_{N, i}\right\rangle\left\langle\Psi_{N, i}\right|
$$

Orthonormal $N$-particle states are denoted by $\left|\Psi_{N, i}\right\rangle$, and the normalized statistical weights, $w_{N, i}$, satisfy $\sum_{N, i} w_{N, i}=1$. One obtains a set of thermal KS equations through a mapping similar to the ZT one, but this time keeping both the equilibrium density and temperature fixed. This defines the XC free energy, which now includes an entropic term. The MerminKohn-Sham (MKS) equations ${ }^{12,22}$ are similar to the groundstate KS equations, but are complicated by temperaturedependent eigenvalues, eigenstates, and effective potential. ${ }^{26}$ The free energy of the physical system is written in the usual way

$$
A=T-\tau S+V_{e e}+V_{\mathrm{ext}},
$$

where $T$ is the kinetic energy, $S$ is the entropy, $V_{e e}$ is the electron-electron repulsion, and $V_{\text {ext }}$ is the external, nuclear potential. ${ }^{50}$ To emphasize the tied coordinate-temperature scaling relationships in thermal DFT, we can write the kentropy $^{25}$ as $K=T-\tau S$. The same equation for the free energy of the systems can be written in terms of the corresponding KS quantities:

$$
A=T_{s}-\tau S_{s}+U+A_{\mathrm{xc}}+V_{\mathrm{ext}},
$$

where the subscript $s$ denotes the KS quantities, $U$ is the Hartree energy, and $A_{\mathrm{xc}}$ is the XC free energy. Mirroring the interacting case, the non-interacting kentropy is written $K_{s}=T_{s}-\tau S_{s}$.

While the operators of quantum mechanics scale in a simple way, the conversion to density functionals invites some 
complexity. One commonly exploited relationship in modern DFT is that between coordinate scaling and the coupling constant. ${ }^{51}$ When the length scale of our system is changed by a factor $\gamma$, we can maintain the normalization of the system via the following definition of the scaled ZT density:

$$
n_{\gamma}(\mathbf{r})=\gamma^{3} n(\gamma \mathbf{r})
$$

Using this scaled density as input, straightforward scaling of energy quantities result, unless the quantities include correlation.

In the adiabatic connection, a coupling constant, $\lambda$, is introduced into the universal functional, ${ }^{34,51}$

$$
F^{\lambda}[n]=\min _{\Psi \rightarrow n}\left\langle\Psi\left|\hat{T}+\lambda \hat{V}_{e e}\right| \Psi\right\rangle
$$

where, again, $\lambda=0$ yields the fictitious $\mathrm{KS}$ system and $\lambda=1$ gives the real, interacting system of interest. For all values of $\lambda$, the density is that of the physical system. The universal functional can be combined with coordinate and density scaling relationships, yielding the relation,

$$
F^{\lambda}[n]=\lambda^{2} F\left[n_{1 / \lambda}\right]
$$

Here, the fully interacting functional evaluated on a scaled density is multiplied by the square of the inverse of the density scaling factor, giving access to the universal functional at a scaled interaction strength. Explicitly known conditions on other energy components can be written under concurrent coordinate and interaction strength scaling, as we will see later. $^{31,34}$

The same concepts applied in ZT simulated scaling can be extended to the finite-temperature adiabatic connection, ${ }^{25,52}$

$$
A_{\mathrm{XC}}^{\tau}[n]=\int_{0}^{1} d \lambda W_{\mathrm{XC}}^{\tau, \lambda}[n],
$$

where here, the adiabatic connection integrand is both temperature- and interaction strength-dependent: $W_{\text {XC }}^{\tau, \lambda}[n]=$ $U_{\mathrm{XC}}^{\tau, \lambda}[n] / \lambda$. An illustration of the adiabatic connection generalized to finite temperatures is shown in Fig. 2. The red arrow indicates the monotonic behavior of the exchange free energy component, $A_{\mathrm{X}}[n]$, which increases with increasing temperature. On the other hand, the correlation free energy component, $A_{\mathrm{C}}$, shows more complex behavior, and varies nonmonotonically as the temperature increases.

\section{METHODS}

When discussing the uniform gas, constraints on the density functions describing the system are similar to those on density functionals $^{34}$ and can be used to extract useful information about properties such as limiting behavior. For instance, coordinate scaling can be expressed in terms of interaction strength scaling in the expression for the $\mathrm{XC}$ free energy:

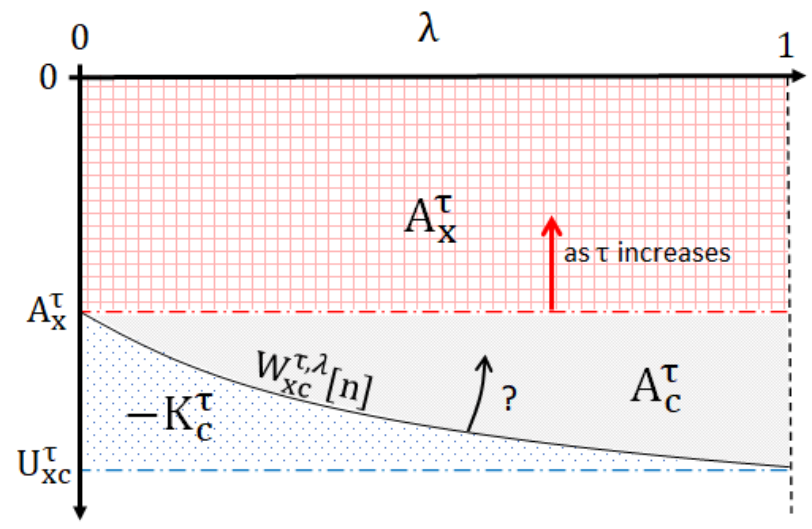

FIG. 2. A diagram of the finite-temperature adiabatic connection. The area in red depicts the exchange free energy component of $A_{\mathrm{XC}}$, the gray area represents the correlation free energy, and $K_{\mathrm{C}}$ is the kentropic correlation, or the FT analog of the kinetic correlation. While the behavior of $A_{\mathrm{X}}$ for a fixed $r_{s}$ with increasing temperature is predictable, the temperature-dependent behavior of $A_{\mathrm{C}}$ is more complex.

$$
a_{\mathrm{xc}}^{\tau, \lambda}(n)=\lambda^{2} a_{\mathrm{xc}}^{\tau / \lambda^{2}}\left(n_{1 / \lambda}\right) .
$$

The exchange free energy contribution, now expressed in terms of the Wigner-Seitz radius, $r_{s}$, can be extracted by scaling to the high-density limit of the FT uniform gas ${ }^{45,53,54}$ :

$$
a_{\mathrm{x}}^{\tau}\left(r_{s}\right)=\lim _{\gamma \rightarrow \infty} \frac{a_{\mathrm{xc}}^{\gamma^{2} \tau}\left(r_{s} / \gamma\right)}{\gamma} .
$$

The expression for the correlation component of the free energy is then obtained using the simple relationship between exchange and correlation:

$$
a_{\mathrm{C}}^{\tau}\left(r_{s}\right)=a_{\mathrm{xc}}^{\tau}\left(r_{s}\right)-a_{\mathrm{x}}^{\tau}\left(r_{s}\right) .
$$

These results of tied coordinate-temperature-interaction strength scaling can be combined to extract any component of the correlation free energy from any other piece. ${ }^{52} \mathrm{We}$ extract the potential contribution from the full correlation free energy via

$$
u_{\mathrm{C}}^{\tau}\left(\gamma, r_{s}\right)=-\gamma \frac{d a_{\mathrm{C}}\left(\frac{r_{s}}{\gamma}, \gamma^{2} \tau\right)}{d \gamma}+2 a_{\mathrm{C}}\left(\frac{r_{s}}{\gamma}, \gamma^{2} \tau\right)
$$

and combine it with the definition of the FT adiabatic connection integrand for the uniform gas,

$$
W_{\mathrm{C}}^{\tau, \lambda}=\frac{u_{\mathrm{C}}^{\tau, \lambda}\left(1 / r_{s}\right)}{\lambda}=\frac{\lambda^{2} u_{\mathrm{C}}^{\tau / \lambda^{2}}\left(\lambda r_{s}\right)}{\lambda}=\lambda u_{\mathrm{C}}^{\tau / \lambda^{2}}\left(\lambda r_{s}\right) .
$$

This yields the finite-temperature adiabatic connection integrand in terms of $a_{\mathrm{C}}^{\tau}\left(r_{s}\right)$. This adiabatic connection formula 
TABLE I. Comparison of the XC free energy values generated using the Fortran 90 program ${ }^{49}$ provided by Groth and collaborators and XC free energy values obtained via integration of simulated scaling curves (FT AC). Improved agreement with the Fortran values can be obtained by increasing the resolution in interaction strength, $\lambda$, in the numerical implementation of simulated scaling.

\begin{tabular}{l|rr|rr|rr}
\hline \hline & \multicolumn{2}{|c|}{$r_{s}=1$} & \multicolumn{2}{c|}{$r_{s}=2$} & \multicolumn{2}{c}{$r_{s}=4$} \\
& Groth et al. & FT AC & Groth et al. & FT AC & Groth et al. & FT AC \\
\hline$\tau=0.1$ & -0.51668 & -0.51667 & -0.27194 & -0.27192 & -0.13375 & -0.13374 \\
$\tau=0.5$ & -0.50031 & -0.50027 & -0.22335 & -0.22335 & -0.08779 & -0.08779 \\
$\tau=1.0$ & -0.45919 & -0.45922 & -0.18167 & -0.18167 & -0.06731 & -0.06731 \\
\hline \hline
\end{tabular}

allows us to calculate the temperature-dependent correlation free energy at any coupling constant strength and temperature. To do so, we use the parametrization in Ref. [24] for the FT UEG and construct finite-temperature adiabatic connection curves at varying density and temperature conditions, using Mathematica Version 11.2. ${ }^{55}$ Table 1 compares values of the XC free energy, obtained from the Fortran 90 code provided by Groth and collaborators, to values obtained using the FTAC formalism. The agreement between the values obtained from the formalism in this work and the Fortran code, and its controllable accuracy via increased interaction strength resolution, verifies the validity of the implemented simulated scaling technique.

Another verification that our application of simulated scaling is sound relies on known behavior of the exchange free energy for the uniform gas. The XC free energy in this work is expressed as a function of the electron degeneracy parameter,

$$
\theta=\frac{2 \tau}{\left(3 \pi^{2} n(r)\right)^{2 / 3}}=2 r_{s}^{2} \tau\left(\frac{4}{9 \pi}\right)^{2 / 3}
$$

The ground-state exchange can be related to $a_{\mathrm{X}}^{\tau}$ via a thermal reduction factor, $R_{\mathrm{X}}$, where $R_{\mathrm{X}}$ can only depend on temperature and density through $\theta$, the electron degeneracy parameter $^{24,26 \text { : }}$

$$
a_{\mathrm{X}}^{\tau}=e_{\mathrm{X}}^{u n i f} R_{\mathrm{X}}(\theta)
$$

The thermal reduction factor decreases with increasing $\theta$, meaning that as the electron degeneracy increases, the exchange free energy decreases in magnitude.

To further validate the use of simulated scaling at finite temperatures, FTAC curves were generated with the same $\theta$ value. These should have the same ratio $W_{\mathrm{XC}}^{\tau, \lambda} / e_{\mathrm{X}}^{\text {unif }}\left(r_{s}\right)$ at $\lambda=0$. Since $\theta$ is a function of $\tau$ and $r_{s}$, individual characteristics can be scaled while the electron degeneracy remains constant. Dividing $W_{\mathrm{XC}}$ by the exact ground-state exchange demonstrates this equivalency. If the electron degeneracy is constant for a set of curves, then the ratio $A_{\mathrm{X}}^{\tau} / e_{\mathrm{X}}^{\text {unif }}$ should also be equivalent for all three curves. As the exchange is constant over interaction strength, plotting the $\mathrm{AC}$ in this manner exposes the correlation component of the XC free energy for pairs of $r_{s}$ and $\tau$ that yield the same electron degeneracy. Fig. 3 shows sets of curves for three different electron degeneracy values, both at low interaction strength and up to $\lambda=1$, the physical interaction strength. These demonstrate the expected behavior at $\lambda=0$. The complicated dependence of correlation on the electron degeneracy arises at relatively low interaction strength, with the set of curves having three times the reference degeneracy crossing those of the $2 \theta_{0}$ set almost immediately, before even a tenth of the physical interaction strength is reached.

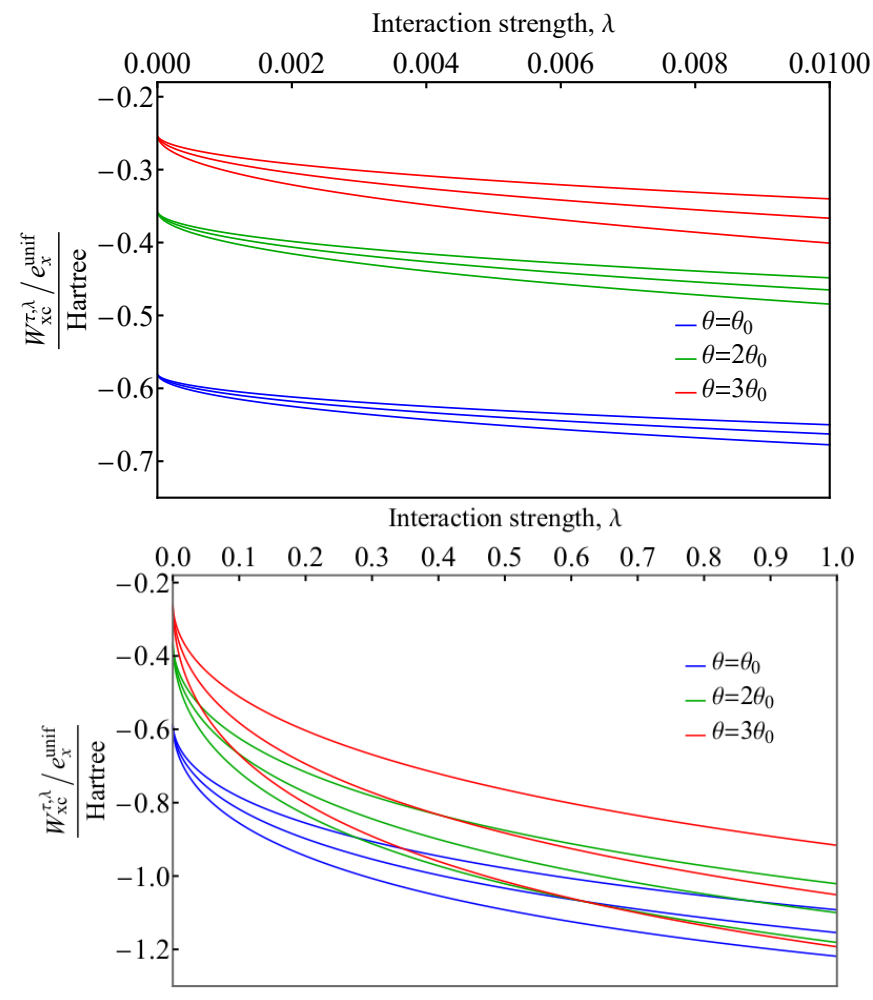

FIG. 3. Plotting curves with equivalent electron degeneracies isolates the mixed temperature-density effect on the correlation free energy. The zoomed-in plot (top) demonstrates how the simple grouping of equivalent electron degeneracy curves is disrupted by correlation effects as the interaction approaches realistic strength (bottom).

\section{RESULTS AND DISCUSSION}

The finite-temperature adiabatic connection (FT AC) curves are observed at low, intermediate, and high temperatures and densities. In dimensionless units, typical parameters 
in the WDM regime are the Wigner-Seitz radius, $r_{s}=r / a_{B}$, and the reduced temperature, $\tau=k_{B} T / E_{F}$, both being of order one for WDM (typically in the range of 0.1-10). Here, $k_{B}$ is the Boltzmann constant, $a_{B}$ is the Bohr radius, and $E_{F}$ is the system's Fermi energy, defined as the zero-temperature limit of the chemical potential. By holding the density fixed as the KS system is smoothly connected to the physical system, the FT ACF demonstrates how the proportions of the exchange and correlation free energy components vary with temperature, density, and interaction strength. In addition, these curves invite investigation of the Lieb-Oxford bound and its relationship to the exchange free energy.

\section{A. Adiabatic Connection Curves}

As an electronic system's temperature rises at a fixed density, it may be expected that the importance of the electronelectron interaction will decrease. However, this oversimplified picture hides the influence of both temperature and density on the components of exchange-correlation. Fig. 4 displays the adiabatic connection curves generated for a uniform gas with moderate density at temperatures varying up to the level of the Fermi energy. The solid curves of the top figure demonstrate the correlation energy while the dashed line corresponds to the exchange component of the highesttemperature curve. For clarity, the exchange component is only explicitly shown for the high-temperature curve, though similar exchange curves could be drawn, starting at $\lambda=0$ for the exchange energy for $\tau=0.1$ and $\tau=0.5$.

The ordering of these curves with rising temperature demonstrates that, at these conditions, the XC free energy does decrease. The bottom plot of Fig. 4, however, isolates the correlation component for all three temperatures. At these conditions, the relative contribution of correlation to $A_{\mathrm{XC}}^{\tau}$ varies greatly with temperature. This is most easily observed in the reversal of the exchange and XC curves' ordering with temperature, but it can also be seen clearly in the flattening of the curves as we move to colder temperatures.

The curves in Fig. 5 demonstrate the adiabatic connection for a uniform gas with a relatively large Wigner-Seitz radius at low, intermediate, and high temperatures. Again, the exchange free energy component is only explicitly shown for the high-temperature curve. Since the Wigner-Seitz radius is inversely proportional to the density, a large Wigner-Seitz radius corresponds to a small density. As expected, the low temperature curve, which was nearly linear in Fig. 4, now exhibits a fairly dramatic decrease at low $\lambda$ values under these conditions. It, and all other calculated curves, obey the exact constraint of a negative first derivative..$^{34,56}$

In contrast to the previous figures, Fig. 6 shows FT ACF curves at a fixed temperature while the density is varied. Here, the XC energy shows similar curvature at all densities when at the Fermi temperature, and the magnitude of the $\mathrm{XC}$ free energy increases with increasing density. This behavior is expected since the magnitude of the exchange energy is inversely related to the Wigner-Seitz radius. The bottom plot of Fig. 6 shows the correlation contribution of each curve

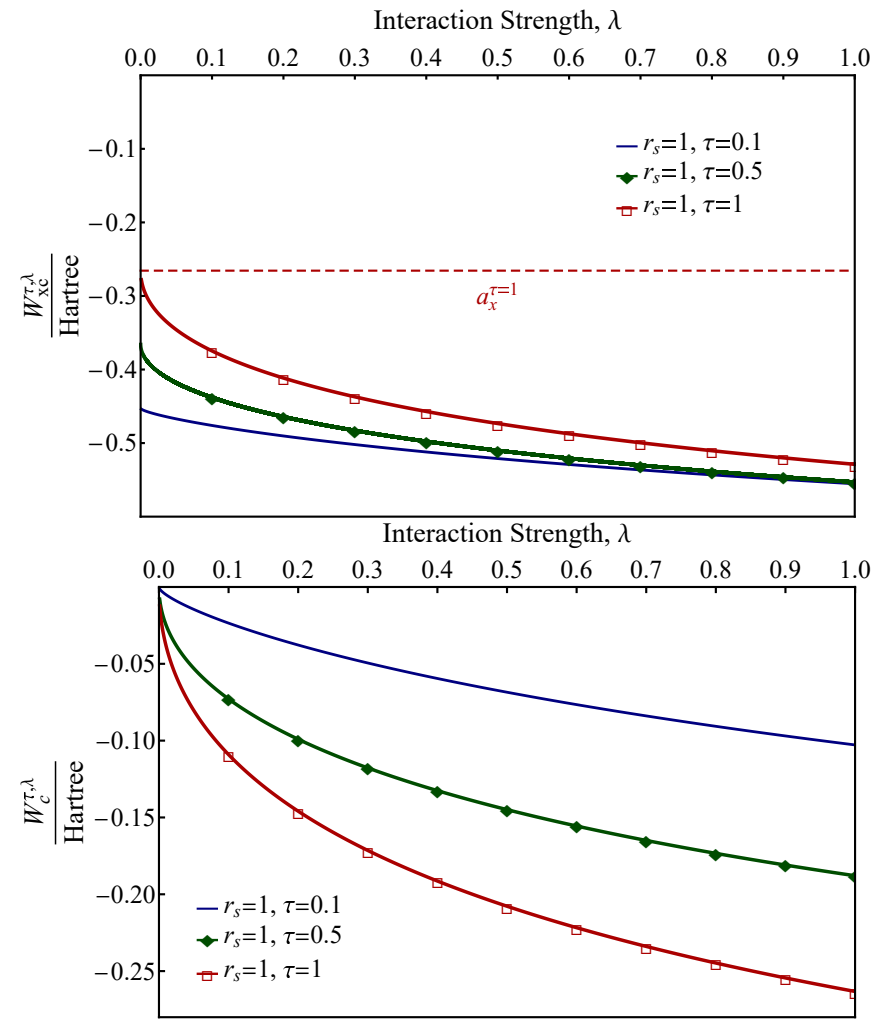

FIG. 4. FT ACF for the UEG at a range of temperatures (top). The exchange (dashed line) is shown for the high-temperature system only. Purely correlation contributions are shown to exhibit opposite ordering with increasing temperature (bottom).

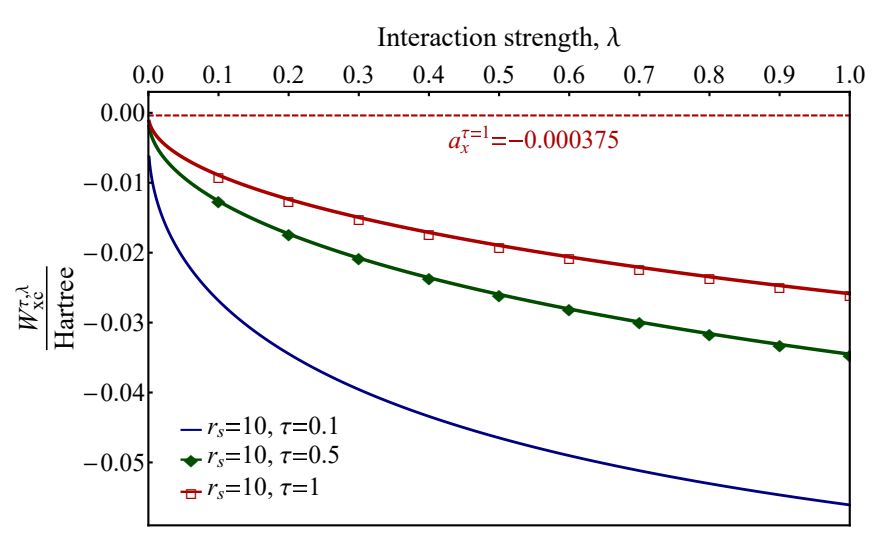

FIG. 5. FT ACF for a low-density UEG is demonstrated for a range of temperatures. The exchange is shown only for the highest temperature. At this high $r_{s}$ value, correlation clearly dominates the XC.

and demonstrates that the behavior of $A_{\mathrm{XC}}^{\tau}$ is dominated by the exchange free energy. Without the exchange component, the ordering of the curves is disrupted at this temperature, and we do not observe the same trend. However, at a high enough temperature, the magnitude of the correlation free energy consistently decreases with increasing $r_{s}$, or decreasing density. The differences in behavior of the correlation free energy at different temperatures demonstrate that the correlation contri- 


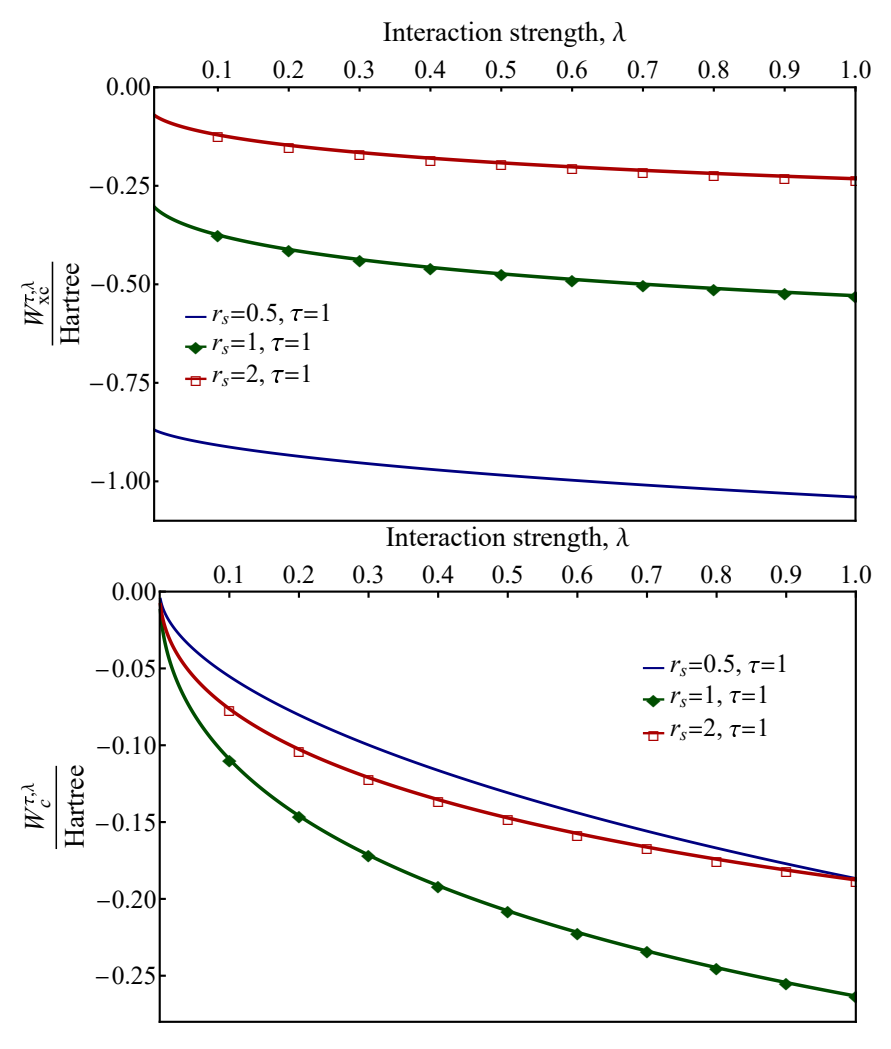

FIG. 6. FT ACF for three different densities at a fixed temperature of $\tau=1$ (top). Purely correlation contributions demonstrate how the ordering of the curves becomes non-monotonic when density is varied at a fixed temperature (bottom).

bution to the XC free energy fluctuates non-monotonically, as predicted via the asymmetric Hubbard dimer. ${ }^{33}$

\section{B. Exact Conditions}

Electron degeneracy, as defined in Eqn.17, can be related to the Lieb-Oxford bound, ${ }^{36,57}$ commonly expressed as an exact inequality for the potential energy contribution to the $\mathrm{XC}$ functional. ${ }^{51} \mathrm{In}$ other words, for wavefunctions that are ground states of an $N$-electron Hamiltonian, the adiabatic connection integrand, $W_{\mathrm{XC}}^{\lambda}$, is limited by the LO bound, where $C_{\mathrm{LO}}$ is the lowest possible number that makes this inequality true for all wavefunctions. ${ }^{42}$ The $\mathrm{LO}$ inequality provides a strict bound on how negative the XC energy can become:

$$
W_{\mathrm{XC}}^{\lambda} \geq-C_{\mathrm{LO}} \int d^{3} r n^{4 / 3}(\mathbf{r}),
$$

where $C_{\mathrm{LO}}$ is a constant. This is often rewritten in terms of the local density approximation to the exchange energy,

$$
W_{\mathrm{XC}}^{\lambda} \geq \frac{-C_{\mathrm{LO}}}{-C_{\mathrm{X}}^{\mathrm{LDA}}} E_{\mathrm{X}}^{\mathrm{LDA}}
$$

where $C_{\mathrm{X}}^{\mathrm{LDA}}$ is the usual constant for the LDA exchange expression. Lieb and Oxford originally established that $C_{\mathrm{LO}} \leq$
1.68 , but these bounds have been tightened by others ${ }^{38,43}$ since: $1.444 \leq C_{\mathrm{LO}} \leq 1.636$.

Some preliminary evidence, based on definitions of the Hartree-exchange energy and low-density limits of $A_{\mathrm{XC}}$, indicates that bounds like the LO bound may be temperaturedependent in thermal DFT. In Fig. 7, ACF curves for a fixed density are shown while the temperature is varied, along with the LO bound and a naive approach to including what might be called implicit temperature dependence. This "quick and dirty" second approach uses the exchange-correlation free energy of our system, $a_{\mathrm{x}}^{\tau}\left(r_{s}\right)$, in place of the zero-temperature $e_{\mathrm{X}}\left(r_{s}\right)$ of the $\mathrm{LO}$ bound expression for the uniform gas:

$$
W_{\mathrm{XC}}^{\lambda} \geq \frac{-C_{\mathrm{LO}}}{-C_{\mathrm{X}}^{\mathrm{LDA}}} e_{\mathrm{X}}^{\text {unif }}\left(r_{s}\right) .
$$

This approach would reduce the integral of Eqn. 19 as temperatures increase for non-uniform systems. At higher densities for the uniform gas, the FT AC curves appear to satisfy this inequality at lower temperatures. However, at a low enough density, $W_{\mathrm{xC}}^{\tau, \lambda}$ is not bound by this attempt to temperatureadjust the LO inequality, even at low temperatures. As shown in Fig. 7, only the true, zero-temperature LO bound is satisfied, whether using the 1999 value for the $C_{\mathrm{LO}}$ constant or the more recently tightened 2019 value. This suggests that, while the LO bound may exhibit some temperature dependence, it is not appropriately captured by the implicit temperature dependence of the thermally weighted density combined with the electron-degeneracy dependence of the thermal reduction factor. ${ }^{24,26}$ In addition, the adherence of the thermal AC curves to the tighter LO bound for these high- $r_{s}$ (i.e., lowdensity) conditions indicate the density-dominated behavior of the low-temperature, low-density limit. Observing this predominately at low densities is related to the well-known dominance of correlation over exchange that holds in finitetemperature systems as well. ${ }^{25}$ Since the exchange comprises a much smaller fraction of the total XC free energy, the correlation is large enough in relative magnitude that it dips down well below the exchange-based dotted curves. As temperatures increase and the curves approach the $\lambda$-axis, it seems likely that inclusion of temperature dependence in the bound could provide a tighter bound than the ZT value, as well as for the full range of $r_{s}$ and $\theta$.

One approach to investigating the temperature-dependence of the LO bound is to look at situations where the LO bound is most likely to hold; for example, when $a_{\mathrm{x}}^{\tau} \ll e_{\mathrm{x}}^{\text {unif }}$. The exchange free energy is most likely to be smaller than the ground state exchange at large values of $\theta$. Since $\theta \propto \tau r_{s}^{2}$, a large value of theta can be achieved with a high value of $\tau$ and low value of $r_{s}$, in which the exchange component of the XC free energy dominates. A large $\theta$ may also be achieved with a low value of $\tau$ and a high value of $r_{s}$, in which the correlation component dominates.

The parameterization provided by Groth and collaborators is valid within $0 \leq \theta \leq 8$ and $0.1 \leq r_{s} \leq 20$. Within this range of $\theta$ values provided by Groth and coworkers, ${ }^{49} W_{\mathrm{xC}}^{\tau, \lambda=0}$ can be as small in magnitude as -0.00092 when $r_{s}=20$ and $\tau \approx 0.1$, and $W^{\tau, \lambda=1}$ can be as low as -4.7780 when $r_{s}=0.1$ and $\tau=0$. Writing the inequality in terms of the uniform gas 


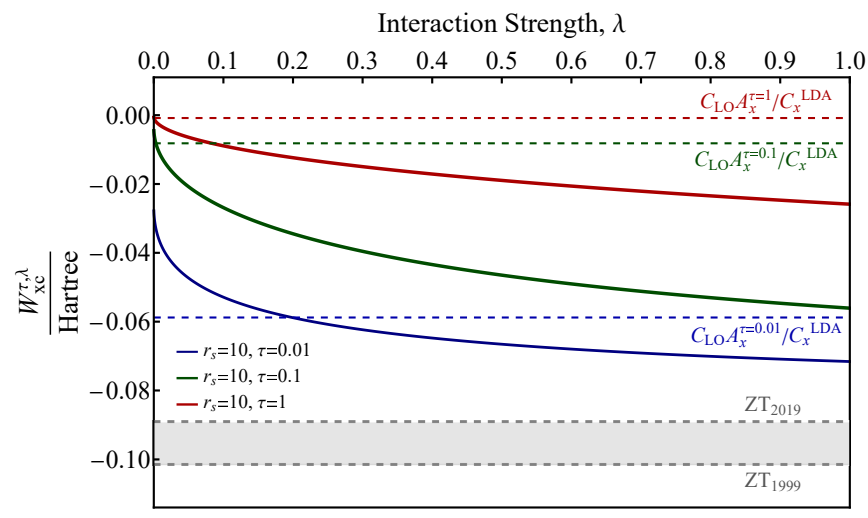

FIG. 7. ZT LO bound and temperature-adjusted quantities for a low density UEG at three different temperatures. Even at low temperatures, the XC free energy is not bounded by the LO-like line calculated from FT exchange. The shaded region indicates the range of possible values of $C_{L O}$ in the Lieb-Oxford inequality.

exchange per particle, ${ }^{42}$

$$
W_{\mathrm{XC}}^{\tau, \lambda} \geq \frac{C_{r_{s}, \lambda}}{C_{\mathrm{X}}^{\mathrm{LDA}}} e_{\mathrm{X}}^{\mathrm{unif}}\left(r_{s}\right)
$$

we define two approximate bounding parameters for our finite-temperature curves, $C_{\text {top }}=C_{r_{s}, \lambda=0}$ and $C_{\text {bottom }}=$ $C_{r_{s}, \lambda=1}$. The $r_{s}$ dependence of these two parameters is rooted in the exchange free energy's dependence on the electron degeneracy, $\theta$, through the reduction factor. This means that maximal and minimal values are constant with respect to $\theta$, but the value shifts depending on how one constructs this degeneracy for a given selection of $r_{s}$ and its corresponding $\tau$ at that specific value of $\theta$.

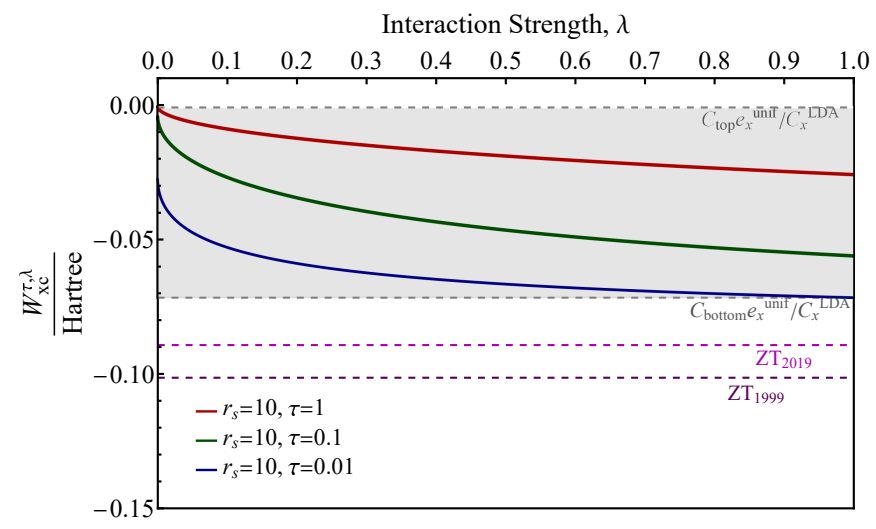

FIG. 8. Bounds are shown for the FTACF at an $r_{s}$ of 10 and three different temperatures. The Lieb-Oxford bound which constrains the exchange-correlation energy at ZT is also shown for the $r_{s}=10$ case.

Figure 8 shows approximated upper and lower bounds for the FTAC for a system of $r_{s}=10$ at three different temperatures. For context, the Lieb-Oxford bound for the ground-state XC energy is also shown, using both the 1999 and 2019 values for $C_{\mathrm{LO}}$. As expected, the ZT bounds are more negative than either of the FT approximated constraints.

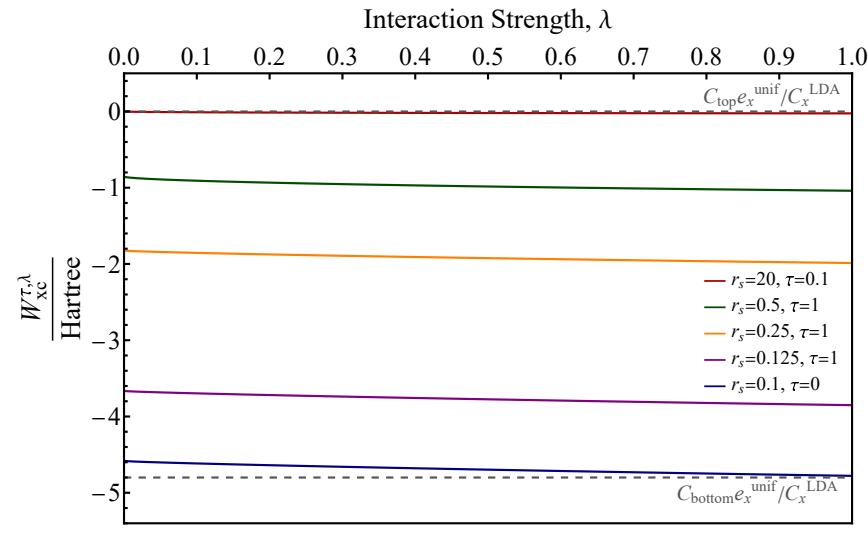

FIG. 9. Approximate upper and lower bounds generated for the FT ACF within the constraints of the parameterization provided by Groth and collaborators are shown for a low electron degeneracy (bottom curve), three intermediate electron degeneracy values, and a large electron degeneracy (top curve).

Figure 9 shows approximated upper and lower bounds for the FT ACF within the allowable range of conditions for the parameterized curves. The curves correspond, from top to bottom, to $\theta=8$, three intermediate electron degeneracies, and $\theta=0$. The curve reaching the highest magnitude corresponds to $\theta=0$, which agrees with the relationship between the exchange free energy and the thermal reduction factor, $R_{\mathrm{X}}(\theta)$. This relationship, along with the dependence of the thermal reduction factor on $\theta$, predicts that the exchange free energy decreases with increasing $\theta$. Thus, when the electron degeneracy is low, the exchange free energy is expected to have a large magnitude, relative to exchange free energy at a high electron degeneracy.

\section{SUMMARY AND FUTURE WORK}

We have presented finite-temperature adiabatic connection curves based on an accurate parameterization ${ }^{49}$ of the exchange-correlation free energy for the uniform electron gas at warm dense matter conditions. ${ }^{49}$ Free energies obtained from the simulated scaling process ${ }^{52,58,59}$ reproduce the directly calculated results ${ }^{49}$ with controllable accuracy. This demonstrates the usefulness of simulated scaling for a wide range of temperatures and densities and also the reliability of the parameterization in applications beyond those for which it was originally designed.

The temperature- and density-dependent AC curves presented in this work behave as expected, in line with the ZT curve for the uniform gas in many ways. However, the relationship between density, interaction strength, and temperature is complex and combinations of conditions exist that can disrupt the naive ordering of curves based on temperature alone. As is well understood in the community, this is due to the dependence of the adiabatic connection integrand on the electron correlation. This is similar to previous calculations using the asymmetric Hubbard dimer ${ }^{28,33}$ and supports care- 
ful use of this model system for DFT analysis. In general, the complicated behavior of the correlation free energy with temperature can be hidden by the exchange free energy, due to its strong, monotonic dependence on the electron degeneracy parameter. For careful examination of the correlation, we have demonstrated that scaling to a correlation-dominated limit can expose these details.

All curves obey the ZT Lieb-Oxford bound, as expected. However, the FT exchange free energy for the UEG does not satisfy the same relationship to this bound that the ZT exchange does, particularly in regimes of large electron degeneracy. Even at low temperatures, in low-density regimes, scaling the FT exchange free energy using the ZT Lieb-Oxford constant fails to contain the ACF curves. In other regimes, where the magnitude of correlation is small, the exchange's dependence on temperature is strong enough to disrupt this ZT relationship. Some approximate bounds on the FT ACF have been generated, demonstrating the tied density-temperature effects on exchange-correlation and showing a large discrepancy between the adiabatic connection curves' most negative values and the ZT LO bound, in line with ZT analysis of similar issues. It has been shown ${ }^{42}$ that uniform densities are not the most challenging for the LO bound at ZT, so using thermal densities near or approaching the strong-interaction limit would be a reasonable approach for better estimating any temperature dependence to be found for the LO bound.

The investigations within this work have all been undertaken within the allowed range of applicability given by Groth and coworkers, save for the initial extraction of the exchange free energy by taking a high-density (i.e., low- $r_{s}$ ) limit. Though this represents a deviation from the recommended usage of the parameterization, our tests using known behavior of the exchange for the UEG with the electron degeneracy demonstrate the robustness to extension beyond the low- $r_{s}$ regime into the "very low" regime. Our group continues work to extend our studies of the interplay between temperature, density, and interaction strength via new approaches approximating these curves and investigating the connections between zero-temperature and finite-temperature exchangecorrelation approximations. Though these methods do not rely on constraints such as the Lieb-Oxford bound, work examining the effect of temperature on bounding relationships is ongoing in hopes that connections between the two areas of study will provide insights into both the entropic contributions to the exchange-correlation free energy and the exchangecorrelation as a whole.

The authors have no conflicts of interest to disclose.

\section{ACKNOWLEDGMENTS}

We are grateful for useful discussions with Drs. Juri Grossi, Sara Giarrusso, and Arnold Kim. This work is supported by the U.S. Department of Energy, National Nuclear Security Administration, Minority Serving Institution Partnership Program, under Award DE-NA0003866. We acknowledge all indigenous peoples local to the site of University of California, Merced, including the Yokuts and Miwuk. We embrace their continued connection to this region and thank them for allowing us to live, work, learn, and collaborate on their traditional homeland.

\section{REFERENCES}

${ }^{1}$ Frank Graziani, Michael P. Desjarlais, Ronald Redmer, and Samuel B. Trickey, editors. Frontiers and Challenges in Warm Dense Matter, volume 96 of Lecture Notes in Computational Science and Engineering. Springer International Publishing, 2014.

${ }^{2}$ Simon Groth, Tobias Dornheim, and Michael Bonitz. Free energy of the uniform electron gas: Testing analytical models against first-principles results. Contrib. Plasma Phys., 57(3):137-146, Mar 2017.

${ }^{3}$ M. D. Knudson and M. P. Desjarlais. Shock compression of quartz to 1.6 TPa: Redefining a pressure standard. Phys. Rev. Lett., 103:225501, Nov 2009.

${ }^{4}$ André Kietzmann, Ronald Redmer, Michael P. Desjarlais, and Thomas R. Mattsson. Complex behavior of fluid lithium under extreme conditions. Phys. Rev. Lett., 101:070401, Aug 2008.

${ }^{5}$ Seth Root, Rudolph J. Magyar, John H. Carpenter, David L. Hanson, and Thomas R. Mattsson. Shock compression of a fifth period element: Liquid xenon to 840 GPa. Phys. Rev. Lett., 105(8):085501, Aug 2010.

${ }^{6}$ M. D. Knudson, M. P. Desjarlais, R. W. Lemke, T. R. Mattsson, M. French, N. Nettelmann, and R. Redmer. Probing the interiors of the ice giants: Shock compression of water to $700 \mathrm{gpa}$ and $3.8 \mathrm{~g} / \mathrm{cm}^{3}$. Phys. Rev. Lett., 108:091102, Feb 2012.

${ }^{7}$ B. Militzer, W. B. Hubbard, J. Vorberger, I. Tamblyn, and S. A. Bonev. A Massive Core in Jupiter Predicted from First-Principles Simulations. Astrophys. J. Lett., 688(1):L45-L48, Oct 2008.

${ }^{8}$ Ralph Ernstorfer, Maher Harb, Christoph T. Hebeisen, Germán Sciaini, Thibault Dartigalongue, and R. J. Dwayne Miller. The Formation of Warm Dense Matter: Experimental Evidence for Electronic Bond Hardening in Gold. Science, 323(5917):1033-1037, Feb 2009.

${ }^{9}$ R. Nora, W. Theobald, R. Betti, F. J. Marshall, D. T. Michel, W. Seka, B. Yaakobi, M. Lafon, C. Stoeckl, J. Delettrez, A. A. Solodov, A. Casner, C. Reverdin, X. Ribeyre, A. Vallet, J. Peebles, F. N. Beg, and M. S. Wei. Gigabar Spherical Shock Generation on the OMEGA Laser. Phys. Rev. Lett., 114(4):045001, Jan 2015.

${ }^{10}$ O. A. Hurricane, D. A. Callahan, D. T. Casey, E. L. Dewald, T. R. Dittrich, T. Döppner, S. Haan, D. E. Hinkel, L. F. Berzak Hopkins, O. Jones, A. L. Kritcher, S. Le Pape, T. Ma, A. G. MacPhee, J. L. Milovich, J. Moody, A. Pak, H.-S. Park, P. K. Patel, J. E. Ralph, H. F. Robey, J. S. Ross, J. D. Salmonson, B. K. Spears, P. T. Springer, R. Tommasini, F. Albert, L. R. Benedetti, R. Bionta, E. Bond, D. K. Bradley, J. Caggiano, P. M. Celliers, C. Cerjan, J. A. Church, R. Dylla-Spears, D. Edgell, M. J. Edwards, D. Fittinghoff, M. A. Barrios Garcia, A. Hamza, R. Hatarik, H. Herrmann, M. Hohenberger, D. Hoover, J. L. Kline, G. Kyrala, B. Kozioziemski, G. Grim, J. E. Field, J. Frenje, N. Izumi, M. Gatu Johnson, S. F. Khan, J. Knauer, T. Kohut, O. Landen, F. Merrill, P. Michel, A. Moore, S. R. Nagel, A. Nikroo, T. Parham, R. R. Rygg, D. Sayre, M. Schneider, D. Shaughnessy, D. Strozzi, R. P. J. Town, D. Turnbull, P. Volegov, A. Wan, K. Widmann, C. Wilde, and C. Yeamans. Inertially confined fusion plasmas dominated by alpha-particle self-heating. Nat. Phys., 12(8):800-806, Apr 2016.

${ }^{11} \mathrm{P}$. Hohenberg and W. Kohn. Inhomogeneous electron gas. Phys. Rev., 136(3B):B864-B871, Nov 1964.

${ }^{12}$ W. Kohn and L. J. Sham. Self-consistent equations including exchange and correlation effects. Phys. Rev., 140(4A):A1133-A1138, Nov 1965.

${ }^{13}$ Thomas R. Mattsson and Michael P. Desjarlais. Phase diagram and electrical conductivity of high energy-density water from density functional theory. Phys. Rev. Lett., 97:017801, Jul 2006.

${ }^{14}$ Winfried Lorenzen, Bastian Holst, and Ronald Redmer. Demixing of hydrogen and helium at megabar pressures. Phys. Rev. Lett., 102:115701, Mar 2009.

${ }^{15}$ M. D. Knudson, M. P. Desjarlais, A. Becker, R. W. Lemke, K. R. Cochrane, M. E. Savage, D. E. Bliss, T. R. Mattsson, and R. Redmer. Direct obser- 
vation of an abrupt insulator-to-metal transition in dense liquid deuterium. Science, 348(6242):1455-1460, Jun 2015.

${ }^{16}$ Bastian Holst, Ronald Redmer, and Michael P. Desjarlais. Thermophysical properties of warm dense hydrogen using quantum molecular dynamics simulations. Phys. Rev. B, 77:184201, May 2008.

${ }^{17}$ M. D. Knudson, M. P. Desjarlais, and Aurora Pribram-Jones. Adiabatic release measurements in aluminum between 400 and $1200 \mathrm{GPa}$ : Characterization of aluminum as a shock standard in the multimegabar regime. Phys. Rev. B, 91(22):224105, Jun 2015.

${ }^{18}$ R F Smith, J H Eggert, R Jeanloz, T S Duffy, D G Braun, J R Patterson, R E Rudd, J Biener, A E Lazicki, A V Hamza, J Wang, T Braun, L X Benedict, $\mathrm{P}$ M Celliers, and G W Collins. Ramp compression of diamond to five terapascals. Nature, 511(7509):330-3, Jul 2014.

${ }^{19}$ Zhandos Moldabekov, Tobias Dornheim, Maximilian Böhme, Jan Vorberger, and Attila Cangi. The relevance of electronic perturbations in the warm dense electron gas. J. Chem. Phys., 155(12):124116, Sep 2021.

${ }^{20}$ Tobias Dornheim, Maximilian Böhme, Zhandos A. Moldabekov, Jan Vorberger, and Michael Bonitz. Density Response of the Warm Dense Electron Gas beyond Linear Response Theory: Excitation of Harmonics. arXiv, Apr 2021.

${ }^{21}$ Zhandos Moldabekov, Tobias Dornheim, Jan Vorberger, and Attila Cangi. Benchmarking Exchange-Correlation Functionals in the Spin-Polarized Inhomogeneous Electron Gas under Warm Dense Conditions. arXiv, Oct 2021.

${ }^{22}$ N. D. Mermin. Thermal properties of the inhomogenous electron gas. Phys. Rev., 137:A: 1441, 1965.

${ }^{23}$ M. W. C. Dharma-wardana and R. Taylor. Exchange and correlation potentials for finite temperature quantum calculations at intermediate degeneracies. J. Phys. C: Solid State Phys., 14(5):629-646, Feb 1981.

${ }^{24}$ François Perrot and M. W. C. Dharma-wardana. Exchange and correlation potentials for electron-ion systems at finite temperatures. Phys. Rev. A, 30(5):2619-2626, Nov 1984.

${ }^{25}$ S. Pittalis, C. R. Proetto, A. Floris, A. Sanna, C. Bersier, K. Burke, and E. K. U. Gross. Exact conditions in finite-temperature density-functional theory. Phys. Rev. Lett., 107:163001, Oct 2011.

${ }^{26}$ Aurora Pribram-Jones, Stefano Pittalis, E.K.U. Gross, and Kieron Burke. Thermal density functional theory in context. In Frank Graziani, Michael P. Desjarlais, Ronald Redmer, and Samuel B. Trickey, editors, Frontiers and Challenges in Warm Dense Matter, volume 96 of Lecture Notes in Computational Science and Engineering, pages 25-60. Springer International Publishing, 2014.

${ }^{27}$ Valentin V. Karasiev, Travis Sjostrom, and S. B. Trickey. Generalizedgradient-approximation noninteracting free-energy functionals for orbitalfree density functional calculations. Phys. Rev. B, 86:115101, Sep 2012.

${ }^{28}$ J. C. Smith, A. Pribram-Jones, and K. Burke. Exact thermal density functional theory for a model system: Correlation components and accuracy of the zero-temperature exchange-correlation approximation. Phys. Rev. B, 93(24):245131, Jun 2016.

${ }^{29}$ Zhandos Moldabekov, Tobias Dornheim, Maximilian Böhme, Jan Vorberger, and Attila Cangi. The relevance of electronic perturbations in the warm dense electron gas. The Journal of Chemical Physics, 155(12):124116, 2021.

${ }^{30}$ J. Harris and A. Griffin. Phys. Rev. B, 11:3669, 1975.

${ }^{31}$ D.C. Langreth and J.P. Perdew. The exchange-correlation energy of a metallic surface. Solid State Commun., 17:1425, 1975.

${ }^{32} \mathrm{O}$. Gunnarsson and B.I. Lundqvist. Exchange and correlation in atoms, molecules, and solids by the spin-density-functional formalism. Phys. Rev. B, 13:4274, 1976.

${ }^{33}$ Justin C. Smith, Francisca Sagredo, and Kieron Burke. Warming Up Density Functional Theory. Springer, Singapore, Nov 2017.

${ }^{34}$ M. Levy and J.P. Perdew. Hellmann-Feynman, virial, and scaling requisites for the exact universal density functionals. shape of the correlation potential and diamagnetic susceptibility for atoms. Phys. Rev. A, 32:2010, 1985.

${ }^{35} \mathrm{M}$. Levy. Density-functional exchange-correlation through coordinate scaling in adiabatic connection and correlation hole. Phys. Rev. A, 43:4637, 1991.

${ }^{36} \mathrm{Mel}$ Levy. Universal variational functionals of electron densities, firstorder density matrices, and natural spin-orbitals and solution of the $v$ representability problem. Proceedings of the National Academy of Sciences of the United States of America, 76(12):6062-6065, 1979.

${ }^{37}$ Elliott H. Lieb and Stephen Oxford. Improved lower bound on the indirect coulomb energy. International Journal of Quantum Chemistry, 19(3):427439, 1981.

${ }^{38}$ Garnet Kin-Lic Chan and Nicholas C. Handy. Optimized lieb-oxford bound for the exchange-correlation energy. Phys. Rev. A, 59:3075-3077, Apr 1999.

${ }^{39}$ Mariana M. Odashima and K. Capelle. How tight is the Lieb-Oxford bound? arXiv, Apr 2007.

${ }^{40}$ Mariana M. Odashima, K. Capelle, and S. B. Trickey. Tightened LiebOxford Bound for Systems of Fixed Particle Number. J. Chem. Theory Comput., 5(4):798-807, Apr 2009.

${ }^{41}$ Mathieu Lewin and Elliott H. Lieb. Improved lieb-oxford exchangecorrelation inequality with a gradient correction. Phys. Rev. A, 91:022507, Feb 2015.

${ }^{42}$ Michael Seidl, Stefan Vuckovic, and Paola Gori-Giorgi. Challenging the Lieb-Oxford bound in a systematic way. Mol. Phys., 114(7-8):1076-1085, Apr 2015.

${ }^{43}$ Mathieu Lewin, Elliott H. Lieb, and Robert Seiringer. Floating wigner crystal with no boundary charge fluctuations. Phys. Rev. B, 100:035127, Jul 2019.

${ }^{44}$ J.P. Perdew. Physica B, 172:1, 1991.

${ }^{45}$ John P. Perdew, Kieron Burke, and Matthias Ernzerhof. Generalized gradient approximation made simple. Phys. Rev. Lett., 77(18):3865-3868, Oct 1996. ibid. 78, 1396(E) (1997).

${ }^{46}$ John P. Perdew, Adrienn Ruzsinszky, Jianwei Sun, and Kieron Burke. Gedanken densities and exact constraints in density functional theory. The Journal of Chemical Physics, 140(18):-, 2014.

${ }^{47}$ Jianwei Sun, John P. Perdew, and Adrienn Ruzsinszky. Semilocal density functional obeying a strongly tightened bound for exchange. Proc. Natl. Acad. Sci. U.S.A., 112(3):685-689, Jan 2015.

${ }^{48}$ Valentin V. Karasiev, James W. Dufty, and S.B. Trickey. Nonempirical semilocal free-energy density functional for matter under extreme conditions. Phys. Rev. Lett., 120:076401, Feb 2018.

${ }^{49}$ Simon Groth, Tobias Dornheim, Travis Sjostrom, Fionn D. Malone, W. M. C. Foulkes, and Michael Bonitz. Ab initio exchange-correlation free energy of the uniform electron gas at warm dense matter conditions. Phys. Rev. Lett., 119:135001, Sep 2017.

${ }^{50}$ A. L. Fetter and J. D. Walecka. Quantum theory of many-particle systems. McGraw-Hill, New York, NY, 1971.

${ }^{51}$ Kieron Burke and friends. The ABC of DFT. In preparation.

${ }^{52} \mathrm{~A}$. Pribram-Jones and K. Burke. Connection formulas for thermal density functional theory. Phys. Rev. B, 93:205140, May 2016.

${ }^{53}$ M. Levy. Int. J. Quantum Chem., S23:617, 1989.

${ }^{54}$ Y. Wang and J. P. Perdew. Phys. Rev. B, 43:8911, 1991.

${ }^{55}$ Wolfram Research, Inc. Mathematica, Version 11.2. Champaign, IL, 2020.

${ }^{56}$ A. M. Teale, S. Coriani, and T. Helgaker. Accurate calculation and modeling of the adiabatic connection in density functional theory. The Journal of Chemical Physics, 132(16):164115, 2010.

${ }^{57}$ Elliott H. Lieb and Stephen Oxford. Improved lower bound on the indirect Coulomb energy. Int. J. Quantum Chem., 19(3):427-439, Mar 1981.

${ }^{58}$ W. Terilla D. Frydel and K. Burke. Adiabatic connection from accurate wavefunction calculations. J. Chem. Phys., 112:5292, 2000.

${ }^{59}$ W. Terilla R.J. Magyar and K. Burke. Accurate adiabatic connection curve beyond the physical interaction strength. J. Chem. Phys., 119:696, 2003. 